\title{
Space Vector Based Synchronized PWM Algorithm for Three Level Voltage Source Inverters: Principles and Application to $V / f$ Drives
}

\author{
Abdul Rahiman Beig
}

arbeig@ieee.org
G.Narayanan

Power Electronics Lab.

Department of Electrical Enginnering

Indian Institute of Science

Bangalore 560012 INDIA

gnar@ieee.org
V.T. Ranganathan

vtran@ee.iisc.ernet.in
Abstract- This paper describes the synchronized PWM techniques based on space vector approach for three level voltage source inverters. The objective is to improve the output waveform of the inverters used in high power applications, where the switching frequency is very low. This is achieved by maintaining the synchronization and half wave symmetry, quarter wave symmetry, threephase symmetry in the PWM waveforms. The principles of achieving synchronization and symmetries are presented. The proposed algorithm is implemented on TMS320F240 based digital controller and applied to V/f drive. The experimental waveforms of the inverter output voltage and motor no load current for different operating eonditions of the drive are presented. The $V_{H T H D}$ of the line voltage and $I_{\text {THD }}$ of the no load current of the motor are computed from the experimental data. The performance measure in terms of the $V_{\text {ITHD }}$ of the line voltage is computed for the entire operating region of the drive for the proposed algorithm and the synchronized SPWM technique. The comparative results show that the proposed method has improved THD over SPWM method. Another significant feature of the proposed algorithm is that the symmetry and synchronization leads to self-halancing of the D.C. bus capacitor voltages.

\section{INTRODUCTION}

Three level inverters are preferred for high power applications since they can achieve double the voltage rating; hence double the power capacity with the semiconductor switches of similar rating as that of two level inverters. Switching frequency $\left(f_{s w}\right)$ of these inverters is limited to low values $(350 \mathrm{~Hz}$ to $1 \mathrm{KHz})$ to reduce the switching losses. Hence the ratio of $f_{s w}$ to the fundamental frequency $f_{f}$ or the pulse number $\left(p=f_{s w} / f_{1}\right)$ is low. Under such circumstances, the output voltage of the inverter will he rich in harmonics. The output voltage must he synchronized with its fundamental component in order to eliminate sub harmonics. In the case of two level inverters, it is shown that in addition to synchronization, preserving half wave symmetry (HWS), quarter wave symmetry (QWS) and three phase symmetry (TPS) in the output voltage will improve the performance of the inverter compared to the PWM methods which do not preserve symmetries [I].

Practical three level inverters employ sine triangle PWM (SPWM) technique because of its simplicity. However the space vector PWM (SVPWM) gives a better D.C. utilization and greater flexibility in selecting the switching states. The purpose of the present work is to develop a simple SVPWM technique, which will result in synchronous PWM waveforms with QWS, HWS and TPS. Hence the output voltages will have improved waveform with reduced harmonics. An important feature of this technique is that this method is simple to implement on digital controllers and has reduced computational requirements compared to the conventional SVPWM technique. The basic principle of the space vector approach to synchronization and symmetry is detailed in section II.

Another major contribution of the proposed PWM technique is that the synchronization and symmetry in the output waveforms ensures that the D.C. link capacitor voltages are balanced over every cycle of the fundamental. This will help in reducing the D.C. link capacitor requirements.

The proposed PWM technique is implemented on TMS320F240 fixed point DSP controller. This technique is applied to VIf drive on experimental prototype consisting of $400 \mathrm{~V}, 10 \mathrm{HP}$, Induction Motor powered from 10KVA,IGBT based three level diode clamp inverter. The experimental results are presented in section III. The performance of the proposed technique is studied over the entire operating range of the drive. A comparison in terms of the weighted total harmonic distortion factor of the output line voltage $\left(V_{\text {WTHD }}\right)$ is given for the proposed technique and SPWM technique. The comparative results reveal the superiority of the present method over SPWM technique in terms of the THD.

\section{PRINCIPLES}

Fig. 1 shows the circuit diagram of three level diode clamp inverter. The space vectors associated with the three level inverters on $\alpha-\beta$ plane are shown in fig.2. The states are defined in Table I. In space vector approach to PWM the reference vector $\bar{V}_{r}$ is sampled at regular intervals $T_{s}$, The sampled reference vector is approximated by time averaging the nearest three vectors $\bar{V}_{x}, \bar{V}_{y}$ and $\bar{V}_{z}$ according to (1)

$$
\bar{V}_{r} T_{s}=\bar{V}_{x} T_{x}+\bar{V}_{y} T y+\bar{V}_{z} T_{z},
$$

where, $T_{x}, T_{y}$ and $T_{z}$ are the intervals of $\bar{V}_{x}, \bar{V}_{y}$ and $\bar{V}_{z}$ respectively and $T,=T_{x}+T y+T_{z}$ 


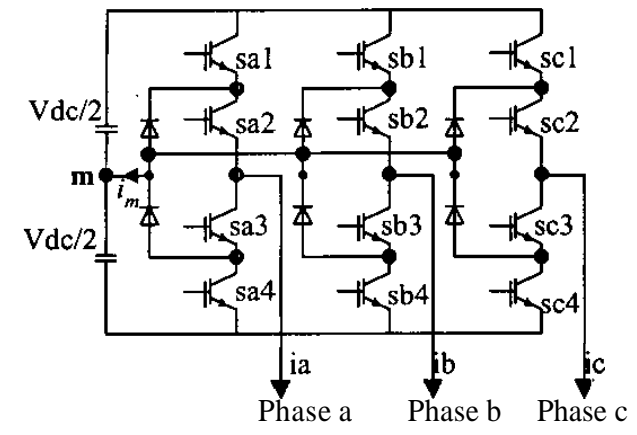

Fig. 1 Circuit digram of three level diode clamp inverter

TABLE I

DEFNITION OF INVERTER STATES

\begin{tabular}{ccc}
\hline State & Switch Position & $\begin{array}{c}\text { Pole voltage with } \\
\text { respect to } V_{\mathrm{m}}\end{array}$ \\
\hline 1 & $\mathrm{sa} 1=\mathrm{ON}, \mathrm{sa} 2=\mathrm{ON}$ & $\mathrm{Vdc} / 2$ \\
& $\mathrm{sa} 3=\mathrm{OFF}, \mathrm{sa} 4=\mathrm{OFF}$ & \\
0 & $\mathrm{sa} 1=\mathrm{OFF}, \mathrm{sa} 2=\mathrm{ON}$ & 0 \\
& $\mathrm{sa} 3=\mathrm{ON}, \mathrm{sa4}=\mathrm{OFF}$ & \\
-1 & $\mathrm{sa} 1=\mathrm{OFF}, \mathrm{sa} 2=\mathrm{OFF}$ & $-\mathrm{Vdc} / 2$ \\
& $\mathrm{sa} 3=\mathrm{ON}, \mathrm{sa} 4=\mathrm{ON}$ & \\
\hline
\end{tabular}

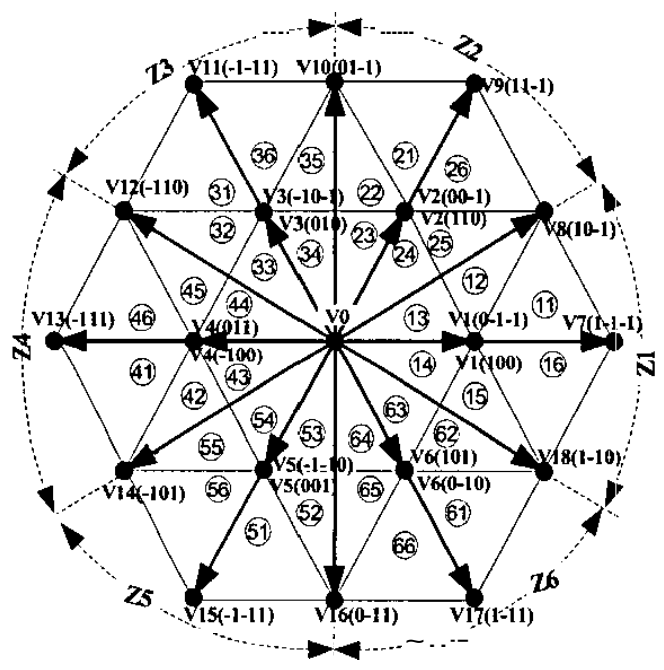

Fig.2. Space vectors of three level inerter.

In order to simplify the above equation, a novel afproach is adopted. The space vector plane is divided in to six sectors, each of sixty degrees interval as shown in fig:!!. Each sector $\mathrm{Z}$, where $\mathrm{Z}=1,2, \ldots 6$, is associated with one pivot vector $\bar{V}_{Z}$ and six other vectors. The pivot vector $\bar{V}_{1}$ and other six vectors of sector 1 are redrawn in fig. 3 (a). The vectors of the other sectors are phase displaced by

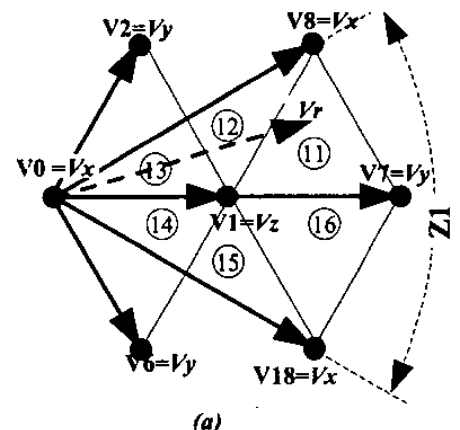

(a)

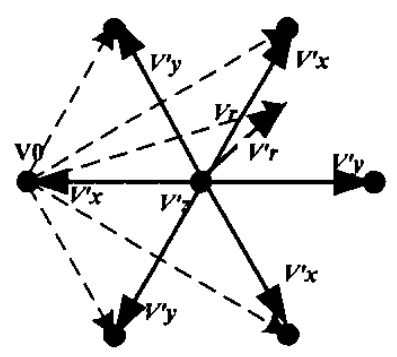

(b)

Fig.3.(a) Space vectors associated with sector 1. (b) Mapping of the vectors of sector Ito fictitious vectors.

TABLE II

PIVOT VECTORS AND THEIR STATES

\begin{tabular}{cccc}
\hline $\begin{array}{c}\text { Sector } \\
Z\end{array}$ & $\begin{array}{c}\text { Pivot Vector } \\
\bar{V}_{z}\end{array}$ & $\bar{V}_{z x}$ & $\bar{V}_{z y}$ \\
\hline 1 & $\bar{V}_{1}$ & 100 & $0-1-1$ \\
2 & $\bar{V}_{2}$ & 110 & $00-1$ \\
3 & $\bar{\nabla}_{3}$ & 010 & $-10-1$ \\
4 & $\bar{V}_{4}$ & 011 & -100 \\
5 & $\bar{V}_{5}$ & 001 & $-1-10$ \\
6 & $\bar{V}_{\leftarrow}$ & 101 & $0-10$ \\
\hline
\end{tabular}

$(Z-1) \pi / 3$ radians. All the six sectors exhibit symmetry and this property is exploited to reduce the computation complexity of the time intervals. All the vectors of a given sector can be mapped to seven vectors, with pivot vector as the origin according to (2).

$$
\begin{aligned}
& \overline{\bar{V}}_{r}^{1}=\bar{V}_{r} e^{j(Z-1) \pi / 3}-\bar{V}_{I}, \\
& \bar{V}_{x}^{1}=\bar{V}_{x} e^{j(Z-1) \pi / 3}-\bar{V}_{1}, \\
& V_{y}^{1} \equiv \bar{V}_{y} e^{j(Z-1) \pi / 3}-\bar{V}_{1} \text { and } \\
& \bar{V}_{z}^{1}=\bar{V}_{z} e^{j(Z-1) \pi / 3}-\bar{V}_{1}=0 .
\end{aligned}
$$


The mapping of the all the seven vectors of sector I to fictitious vectors is illustrated in fig. 3(b). The equation 1 will have only two unknowns as in (3).

$$
\vec{V}_{r}^{1} T_{s}=\bar{V}_{x}^{1} T_{x}+\bar{V}_{y}^{1} T_{y}
$$

The $T_{x}, T_{y}$ and $T_{z}$ are computed using (4),(5) and (6).

$$
V_{r \alpha} T_{s}=\bar{V}_{x \alpha}^{1} T_{x}+\bar{V}_{y \alpha}^{1} T_{y}
$$

$\bar{V}_{r \beta}^{1} T_{s}=\bar{V}_{x \beta}^{1} T_{x}+\bar{V}_{y \beta}^{1} T_{y}$

$T_{z}=T,-T_{X}-T Y$

\section{A. Space Vector Approach to Synchronization and Symmetry}

The pivot vectors can he synthesized by two inverter states, $\bar{V}_{z x}$ and $\bar{V}_{z y}$ as given in Table Il. Switching sequence using all the four space vectors, $\bar{V}_{z x} \Leftrightarrow \bar{V}_{x} \Leftrightarrow \bar{V}_{y} \Leftrightarrow \bar{V}_{z y}$ can he generated, where $T_{z}$ interval is equally split between $\bar{V}_{z x}$ and $\bar{V}_{z y}$. In order to maintain minimum switching frequency the sequences must satisfy following two conditions.

- Condition 1: Only one switch is switched during state transition. That is transition from state 1 to state -1 and vice versa is not allowed.

- Condition 2: The final state of present sample will he the initial state of next sample.

At low switching frequencies, it is necessary to maintain perfect synchronization of inverter output voltage with respect to its own fundamental to avoid sub harmonics. The three-phase symmetry will ensure that all the harmonics and the fundamental of all the three phases will he perfectly balanced. So the triplen harmonics will he cancelled from the line voltage. The half wave symmetry will ensure elimination of even harmonics from the output voltage. The conditions of waveform symmetry for a given sample $\boldsymbol{k}$, are given in first row of the Table III. The necessary and sufficient conditions in terms of the inverter states to achieve synchronization and symmetry at the line and pole voltages are given in the

TABLE III,

CONDITIONS FOR HWS, TPS AND

\begin{tabular}{|c|c|c|c|c|c|}
\hline & $\theta$ & $\begin{array}{c}\theta \pm 360 \\
\text { (Synchron } \\
\text { ization) }\end{array}$ & $\begin{array}{c}\theta \pm 180 \\
\text { HWS }\end{array}$ & $\begin{array}{c}\theta+120 \\
\text { TPS }\end{array}$ & $\begin{array}{c}\theta+240 \\
\text { TPS }\end{array}$ \\
\hline \multirow{3}{*}{ I } & $v a$ & $v a$ & $-v a$ & $v c$ & $v b$ \\
\hline & $v b$ & $v b$ & $-v b$ & $v a$ & $v c$ \\
\hline & $v c$ & $v c$ & $-v c$ & $v b$ & vo \\
\hline & $\mathrm{Sa}$ & $\mathrm{Sa}$ & $\mathrm{S}_{\mathrm{a}} \mathrm{a}$ & $\mathrm{SC}$ & $\mathrm{Sb}$ \\
\hline 2 & Sh & Sh & $S^{\prime} b$ & $\mathrm{Sa}$ & $\mathrm{s} \mathrm{c}$ \\
\hline
\end{tabular}
SYNCHRONIZATION second row of Table III. In table III, 8 is any arbitrary angle measured from the $\boldsymbol{a}$ axis and the states $S_{a}^{\mathrm{l}} S_{b}^{\mathrm{i}} S_{c}^{1}$ indicate the complementary states of $S_{a} S_{b} S_{c}$ (Complementary state of 1 is state -1 and vice versa and complementary state of 0 is state 0 ). The consideration of symmetry demands that there should he integral number of samples $\mathrm{N}$, in each sector of 60 degrees duration and these samples should he positioned at identical intervals at each sector. If $\mathrm{N}$ is odd, then the $N^{\text {th }}$ sample will be on the sector boundary and all other $N-1$ samples are placed at an interval of $\Delta \theta=\frac{\pi / 3}{\mathrm{~N}}$ in a sector. All the samples except the sample during sector change over will have the sequence $\bar{V}_{z x} \rightarrow \bar{V}_{x} \rightarrow \bar{V}_{y} \rightarrow \bar{V}_{z y}$ or $\bar{V}_{z y} \rightarrow \bar{V}_{y} \rightarrow \bar{V}_{x} \rightarrow \bar{V}_{z x}$ alternatively.

During sector change over, the pivot vector $\bar{V}_{z}$ has to he changed accordingly. If $\mathrm{N}$ is odd, the $N^{\text {th }}$ sample will have sequences $\quad \bar{V}_{z x} \Leftrightarrow \bar{V}_{x} \Leftrightarrow \overline{\boldsymbol{v}}, \Leftrightarrow \bar{V}_{z y} \Leftrightarrow \overline{\boldsymbol{v}}, \quad$ or $\bar{V}_{z x} \Leftrightarrow \bar{V}_{x} \Leftrightarrow \bar{V}$, . The former will result in pulse number of $p=(3 N+1) / 2$ and the latter will result in $p=(3 N-1) / 2$. So for odd values of $N=1,3,5 \ldots, p$ will take values $2,5,8 \ldots$ or $1,4,7 \ldots$ If $\mathrm{N}$ is even then there will not he sample on sector boundary and the first and last sample in a sector will have the sequences $\bar{V}_{z x} \Leftrightarrow \bar{V}_{x} \Leftrightarrow \bar{V}_{y} \Leftrightarrow \bar{V}_{x}$. For even values of $\mathrm{N}, \boldsymbol{p}$ is given by $\boldsymbol{p}=(3 N) / 2$. So for $N=$ $2,4,6 \ldots, p$ will take values $3,6,9, \ldots$ Hence for any integer value of pulse number $p$, synchronization and QWS, HWS and TPS will he maintained. So the switching frequency variation in variable frequency drives is small and will result in smooth operation of the drive. This is a significant improvement over synchronized SPWM, where $\boldsymbol{p}$ must be odd multiple of 3 to avoid odd harmonics and triplen harmonics from the line voltage.

\section{B. Implementation}

The reference vector is sampled at $T_{S}=\mathrm{I} /\left(f_{1}{ }^{*} 6^{*} \mathrm{~N}\right)$ and it decides the sector $Z$. Once $Z$ is known then the sample can be mapped to $\bar{V}_{r}^{1}$ as explained above. Also the $Z$ will decide the starting vector $\overline{V_{Z}}$. The information on $\mathrm{N}$ ( $N$ is odd or even) will decide the sequence. An interesting feature of this PWM technique is that the output waveforms will have QWS, that is, $v_{a}\left(\theta_{a}+\#\right)=v_{a}\left(\theta_{a}-\theta\right) \quad v_{b}\left(\theta_{b}+\#\right)=v_{b}\left(\theta_{b}-\theta\right)$ and $v\left(\theta_{c}+\theta\right)=v_{c}\left(\theta_{c}-\theta\right)$, where $\theta_{a}, \theta_{b}$ and $\theta_{c}$ are the angles for which the phase voltages $v_{a}, v_{b}$ and $v_{c}$ are at peak values. This is because the switching sequences satisfy the following condition of QWS, $S_{a}\left(\theta_{a}+\#\right)=S_{a}\left(\theta_{a}-\theta\right)$, $S_{b}\left(\theta_{b}+\theta\right)=S_{b}\left(\theta_{b}-\theta\right)$ and $S_{c}\left(\theta_{c}+\theta\right)=S_{c}\left(\theta_{c}-\theta\right)$. 

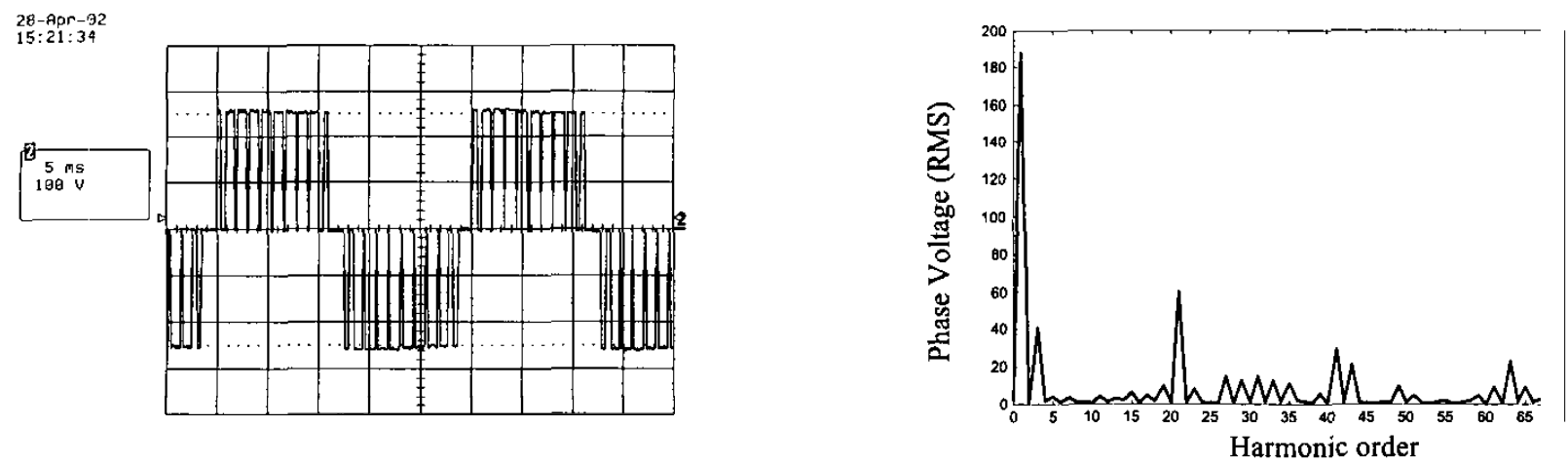

4(d)
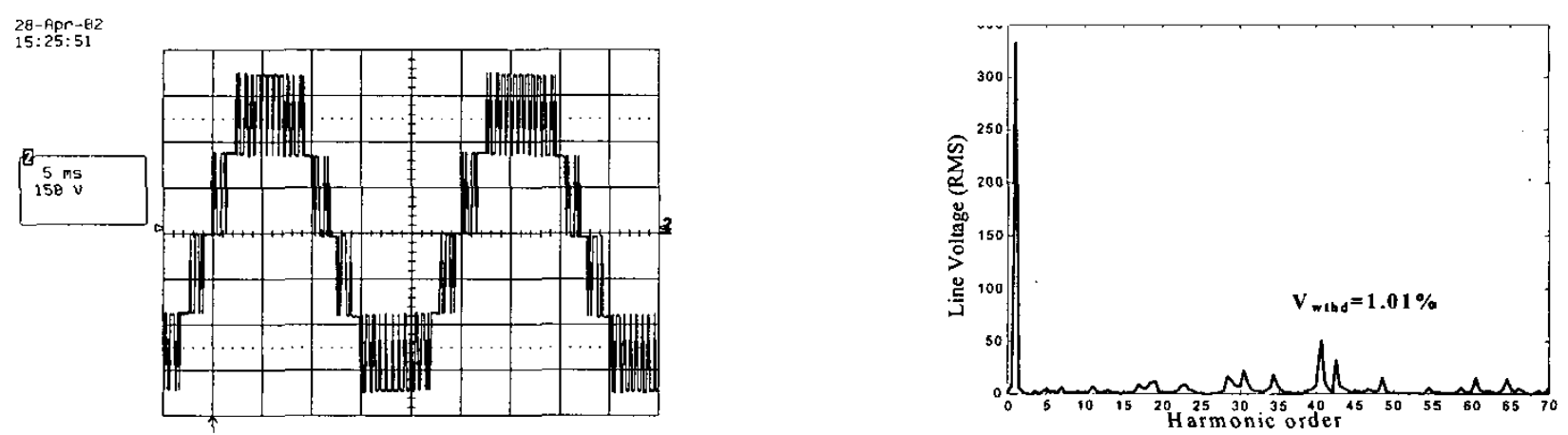

$4(e)$
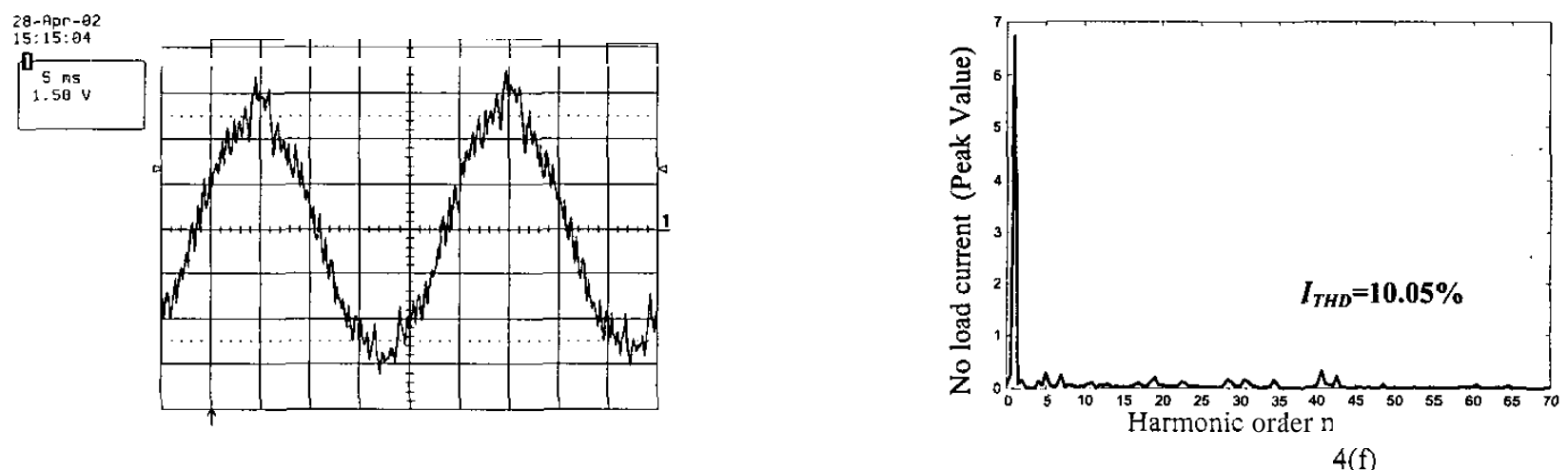

Fig .4. Experimental waveforms: $f_{1}=40 \mathrm{~Hz}, N=7, p=10, f_{s w}=400 \mathrm{~Hz} . V_{l}=320 \mathrm{~V}$ (a) Pole Voltage (b) Line Voltage (c) No load current of the motor. (Scale: $1 \mathrm{~V}=2.5 \mathrm{~A}$ ) (d) Frequency Spectra of Phase voltage, (e) Frequency Spectra of line voltage and (f) Frequency spectra of no load current of the motor

\section{Self Balancing of D.C. Bus Capacitor Voltages}

Fig. 1 shows the power circuit of three level diode clamp inverter. The D.C. link mid point voltage $\left(\nu_{m}\right)$ imbalance is decided by the mid point bus current $i_{m}[3][4]$. Every :sample except the $N^{\text {th }}$ sample will switch $\bar{V}_{z x}$ and $\bar{V}_{z y}$ for equal duration of $T z / 2$ seconds. The $i_{m}$ due to $\bar{V}_{z x}$ is equal and opposite of that of $\bar{V}_{z y}$. So the D.C. link mid point current is cancelled to some extent in every sub cycles. For a given sample $k$ where $k=1,2 \ldots N$, the time intervals $T_{x}(k), T_{y}(k)$ and $T_{z}(k)$ of all the sectors will be identical. So the D.C. Link capacitor voltages are perfectly balanced over every cycle of the fundamental. It can also be shown that under unity power 

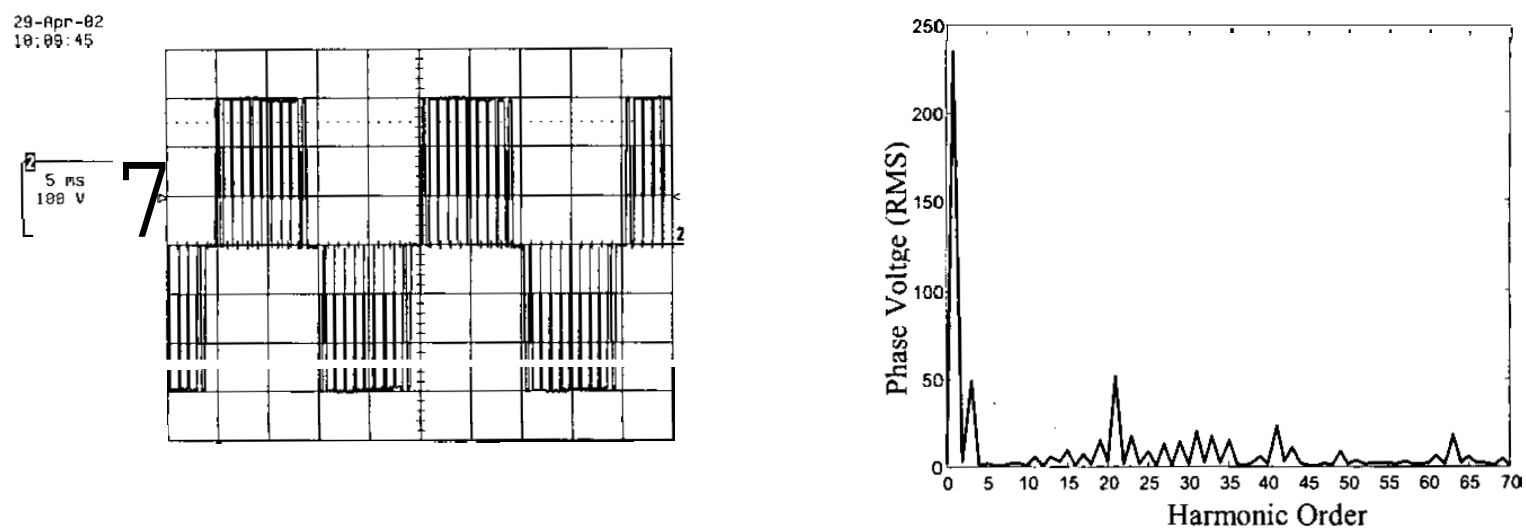

$5(d)$
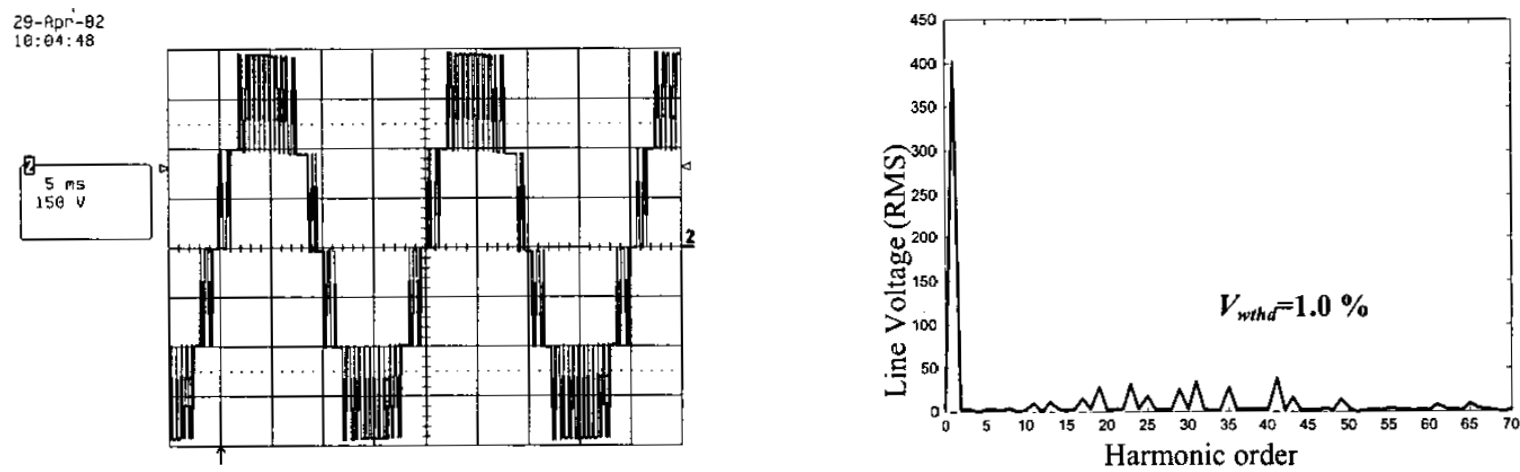

$5(\mathrm{e})$
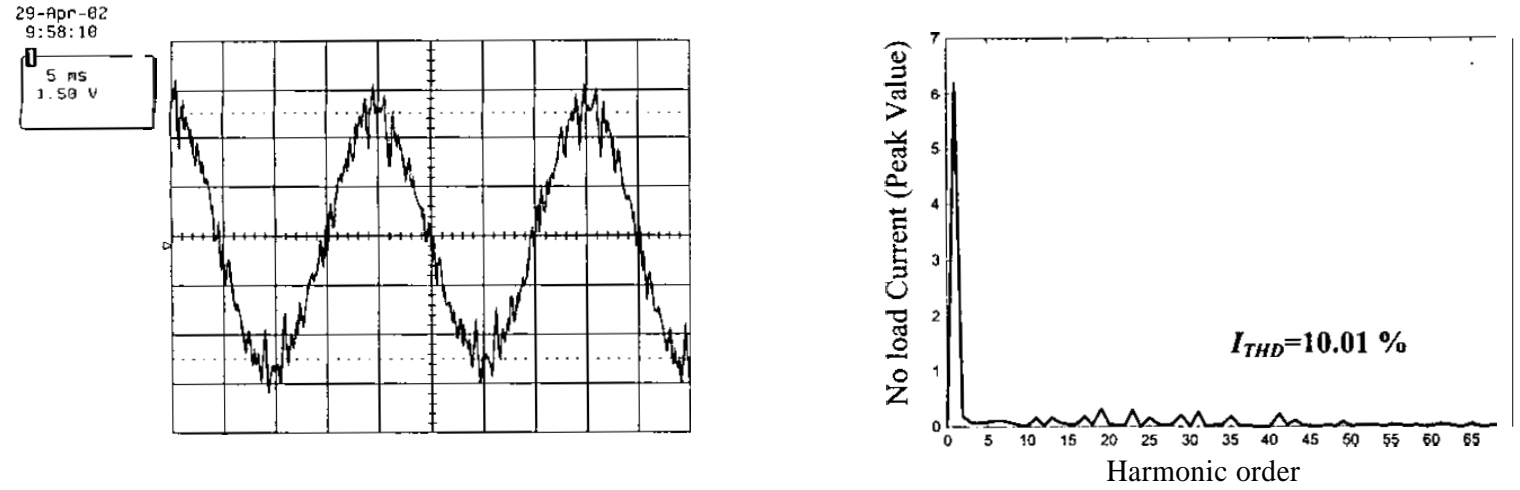

$5(f)$

Fig. 5 Experimental Waveforms: $f_{i}=50 \mathrm{~Hz}, N=7, p=10, f_{s w}=500 \mathrm{~Hz}, V_{j}=400 \mathrm{~V}$ (a) Pole Voltage, (b) Line Voltage (c) No load current of the motor (Scale: $1 \mathrm{~V}=2.5 \mathrm{~A}$ ) (d) Frequency spectra of phase voltage (e) Frequency spectra of line voltage (f) Frequency spectra of the no load current of the motor

factor conditions, the D.C. link capacitor voltages are perfectly balanced on every $(1 / 6)^{t h}$ cycle of the fundamental. Hence the D.C. link capacitor voltage balancing is built in to the algorithm and does not require any additional computation as given in [3]. The D.C. link capacitor requirements can be reduced

\section{EXPERIMENTAL RESULTS}

The proposed algorithm is implemented on TMS 320 F240 fixed point DSP controller. The algorithm is applied to $\mathrm{V} / \mathrm{f}$ drive consisting of $400 \mathrm{~V}, 10 \mathrm{HP}$ induction motor, powered 


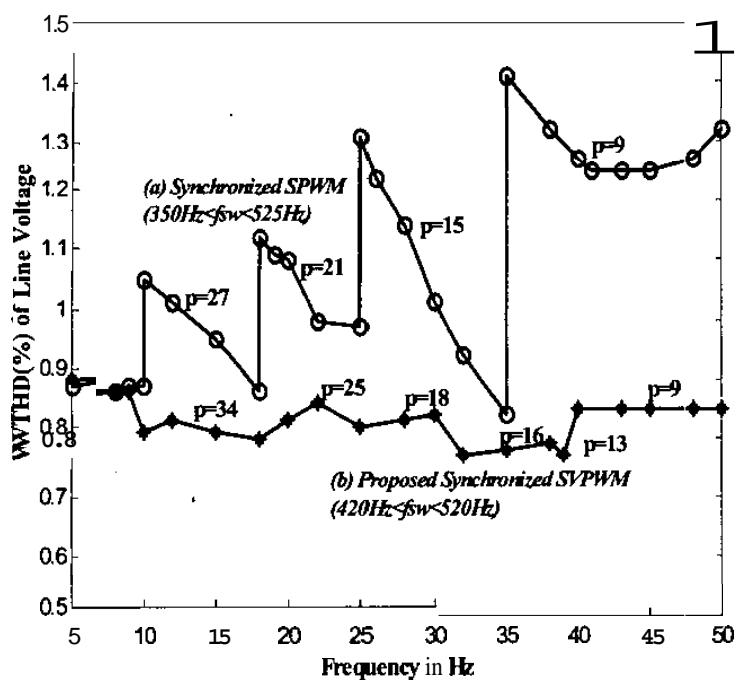

Fig. 6. $V_{\text {wthd }} \mathrm{V} / \mathrm{S} f_{l}$ plot.

from a 30KVA, IGBT based three level diode clamp inverter. The D.C. link voltage is maintained at $500 \mathrm{~V}$ for the linear range of the modulator. The maximum frequency for which the modulator remains in linear range corresponds to $f_{1}=$ $43.3 \mathrm{~Hz}$. For $50 \mathrm{~Hz}$, the D.C. link voltage is maintained at $575 \mathrm{~V}$, which will result in fundamental voltage $\left(V_{l}\right)$ of $400 \mathrm{~V}$, which corresponds to the highest modulation with linear range. The pole voltage, line voltage and the no load current for $f_{1}=40 \mathrm{~Hz}$ and $50 \mathrm{~Hz}$ and their frequency spectra are given in fig. 4 and fig.5 respectively. The $I_{T H O}$ is computad from the experimental data and is $10.05 \%$ and $10.01 \%$ for $f_{l}=40 \mathrm{~Hz}$ and $50 \mathrm{~Hz}$ respectively. Similarly the $V_{W T H D}$, computad from the experimental data for these two conditions is $1.01 \%$ and $1.0 \%$ respectively. The frequency spectra of the line voltage and current demonstrate the absence of sub-harmonics, even harmonics and triplen harmonics for any integer value of pulse number, $p$.

\section{PERFORMANCE ANALYSIS}

In high power drives the total harmonic distortion of the no load current $\left(I_{T I I D}\right)$ of the motor, which is a function of voltage spectra and motor parameters is a suitable performance index at low switching frequencies [1]. $I_{T I D}$ is givenhy $I_{T H D}-\frac{\sqrt{\Sigma I_{n}^{2}}}{I_{1}}, n \neq 1$, where $I$, is the rms value of $\mathrm{n}^{\text {th }}$ harmonic and $I$, is the RMS value of the fundamental component of the no load current of the motor. .Another equivalent quantity, which is a measure of $I_{T I D D}$, function of voltage spectra only and independent of load is the $V_{\text {WTIID }}$ given by $\mathrm{V}_{\boldsymbol{W T H D}}=\frac{\sqrt{\Sigma(V n / n)^{2}}}{V_{1}}, n \neq 1[1],[7]$. In this paper the proposed synchronized PWM technique and the synchronized SPWM are evaluated in terms of the $V_{\text {WTIID }}$.
The $V_{\text {HTHD }}$ is computed from the simulation results for the entire range off, for the proposed method and SPWM. In the case of SPWM synchronization is maintained and pulse number, $p$ is odd integral multiple of 3 . The plot of $V_{M T H D} \mathrm{~V} / \mathrm{s}$ $f_{l}$ is given in fig. 6. The proposed method has improved $V_{W T I D}$ compared to that of SPWM for the entire region. This demonstrates the superiority of the proposed method over the SPWM technique in terms of $V_{\text {WTHD }}$.

\section{CONCLUSIONS}

The theory of the space vector based synchronized PWM method with HWS, QWS and TPS is explained. The experimental results are presented. The proposed algorithm is simple to implement on digital controllers and does not add to any computational complexity. It is shown that the proposed PWM technique will result in balanced D.C. link capacitor voltages. The performance results show that the $V_{\text {WTHD }}$ of the proposed method is better compared to that of SPWM technique. The experimental waveforms show QWS, HWS and TPS for any integer value of pulse number. The absence of triplen harmonics from the line voltage shows that the inverter output voltages have three-phase symmetry. Even though the focus is on low switching frequency applications, the proposed technique can be used for high switching frequency applications also.

\section{REFERENCES}

[I] G.Narayanan and V.T.Ranganathan, "Synchronized PWM strategies based on space vector approach. Part 1. and Part 2, "IEE Proc.Electric Power Applications, vol. 146, no. 3, May 1999.pp 276-289

[2] J. K. Steinke, "Switching Frequency Optimal PWM control of a three level inverter", IEEE Transactions on Power Electronics, vol.7, no 3, July 1992,pp487-496.

[3] H.Y.Liu, N.S.Choi and G.H.Cho, "DSP based space vector PWM for three level inverter with DC link voltage balancing.", Proceedings of the IECON9I conference, Sept. 1991, pp 197-203.

[4] N. Celanovic and D. Borojevic, "A comprehensive study of Neutral-Point voltage balancing problem in three level Neutral Point Clamped voltage source PWM Inverters", IEEE Transactions on Power Electronics, vol. 15, no 2, March 2002, pp 242 -249.

[5] G.Carrara, S.Gardella, M.Marchesoni, R.Salutari and G.Sciutto, "A new multilevel PWM method: A Theoretical analysis", IEEE Transaction on Power Electronics, vol.7, no.5, July 1992, pp 497-505.

[6] H.Stemmler, "High-power industrial drives ", Proc. IEEE, vol 82, Aug.1994, pp. 1266-1286.

[7] M.Hava, R.J.Kerkaman and T.A. Lipo, "Carrier based PWM-VSI over modulation strategies: Analysis, comparison and design," IEEE Transactions on power Electronics, vol.13, no.4, July 1998, pp 674-680.

[8] J.Holtz " "Pulse width modulation - a survey". IEEE transactions on Industrial Electronics, vol. 39 no.5, 1992,pp410 -420 . 